\title{
Identification and analysis of evolutionary selection pressures acting at the molecular level in five forkhead subfamilies Christina D Fetterman*1, Bruce Rannala ${ }^{2}$ and Michael A Walter ${ }^{1}$
}

Address: ${ }^{1}$ Department of Medical Genetics, University of Alberta, Edmonton, Alberta, Canada and ${ }^{2}$ Genome Center and Section of Evolution and Ecology, University of California, Davis, California, USA

Email: Christina D Fetterman* - christina.fetterman@ualberta.ca; Bruce Rannala - brannala@ucdavis.edu; Michael A Walter - mwalter@ualberta.ca

* Corresponding author

Published: 24 September 2008

BMC Evolutionary Biology 2008, 8:26I doi:10.1|86/|47|-2|48-8-26|
Received: 25 February 2008

Accepted: 24 September 2008

This article is available from: http://www.biomedcentral.com/|47|-2|48/8/26I

(c) 2008 Fetterman et al; licensee BioMed Central Ltd.

This is an Open Access article distributed under the terms of the Creative Commons Attribution License (http://creativecommons.org/licenses/by/2.0), which permits unrestricted use, distribution, and reproduction in any medium, provided the original work is properly cited.

\begin{abstract}
Background: Members of the forkhead gene family act as transcription regulators in biological processes including development and metabolism. The evolution of forkhead genes has not been widely examined and selection pressures at the molecular level influencing subfamily evolution and differentiation have not been explored. Here, in silico

pressures acting on the coding sequence of five multi-species FOX protein subfamily clusters; FoxA, FoxD, Foxl, FoxO and FoxP.

Results: Application of site models, which estimate overall selection pressures on individual codons throughout the phylogeny, showed that the amino acid changes observed were either neutral or under negative selection. Branch-site models, which allow estimated selection pressures along specified lineages to vary as compared to the remaining phylogeny, identified positive selection along branches leading to the FoxA3 and Protostomia clades in the FoxA cluster and the branch leading to the FoxO3 clade in the FoxO cluster. Residues that may differentiate paralogs were identified in the FoxA and FoxO clusters and residues that differentiate orthologs were identified in the FoxA cluster. Neutral amino acid changes were identified in the forkhead domain of the FoxA, FoxD and FoxP clusters while positive selection was identified in the forkhead domain of the Protostomia lineage of the FoxA cluster. A series of residues under strong negative selection adjacent to the $\mathrm{N}$ - and $\mathrm{C}$-termini of the forkhead domain were identified in all clusters analyzed suggesting a new method for refinement of domain boundaries. Extrapolation of domains among cluster members in conjunction with selection pressure information allowed prediction of residue function in the FoxA, FoxO and FoxP clusters and exclusion of known domain function in residues of the FoxA and Foxl clusters.

Conclusion: Consideration of selection pressures observed in conjunction with known functional information allowed prediction of residue function and refinement of domain boundaries. Identification of residues that differentiate orthologs and paralogs provided insight into the development and functional consequences of paralogs and forkhead subfamily composition differences among species. Overall we found that after gene duplication of forkhead family members, rapid differentiation and subsequent fixation of amino acid changes through negative selection has occurred.
\end{abstract}




\section{Background}

A highly conserved DNA binding domain, termed 'forkhead' due to the physical appearance of Drosophila fork head mutants, defines forkhead gene family members. Forkhead family members act as transcription activators or repressors in biological processes involved in development and metabolism. Human diseases such as AxenfeldRieger syndrome [1], lymphedema-distichiasis [2], developmental verbal dyspraxia [3], and various cancers [4-7] have been associated with mutations or chromosomal rearrangements of forkhead genes. Forkhead genes have been identified in a wide variety of animals and fungi but not plants. Within the forkhead gene family, subfamilies were delineated by their position within a phylogenetic tree that was created using only the forkhead domain sequences [8]. Different subfamilies are identified by letters, with subfamilies A through S noted in humans. For many species, multiple members of a subfamily are known to exist and are further delineated by Arabic numerals.

While some research has examined forkhead gene family evolution, selection pressures on individual codons have not been measured and studies that have examined evolutionary forces acting on entire forkhead genes have included only orthologous sequences from a subfamily. Here we analyze entire subfamilies to explore the evolutionary and functional significance of subfamily paralogs and orthologs. Gene duplication, and subsequent selection driving adaptive evolution, is thought to create gene families with differentiated family members. At the molecular level, amino acid changes that result in reduced fitness are removed by negative selection whereas changes that increase fitness are maintained by positive selection. When amino acid changes do not decrease or increase fitness, the changes are considered neutral. At individual codons, also known as sites, natural selection can be measured in terms of $\omega$, the nonsynonymous substitution rate divided by the synonymous substitution rate. An $\omega<$ 1 indicates negative selection is occurring while $\omega>1$ suggests positive selection and $\omega=1$ for neutral changes. Negative or positive selection of amino acid residues implies that the residues are functionally important. Neutral changes at amino acid sites imply that the exact composition of amino acids at these sites is unimportant and that they are not directly involved in protein function.

We sought to identify the selection pressures acting on individual amino acid sites in forkhead gene family members. Five forkhead subfamilies, FoxA, FoxD, FoxI, FoxO and FoxP were examined independently using branch-site and site models implemented in the codeml program, contained in the PAML package. The results of our analysis of site and lineage specific selection patterns, in conjunction with prior information concerning the functional importance of amino acid residues in each cluster, provide insights into forkhead gene family evolution and information regarding potential functional and nonfunctional amino acids in this important transcription factor gene family.

\section{Methods \\ Sequence Data}

A list of 672 amino acid sequences containing the forkhead domain was retrieved from the NCBI Entrez Protein Database using the Conserved Domain Architecture Retrieval Tool (CDART) [9] in conjunction with the Conserved Domain Database forkhead domain definition, cd00059 [10,11]. Sequences described as partial, incomplete, fragment, predicted, putative and hypothetical as well as duplicates and isoforms were excluded resulting in a total of 299 sequences from 51 species analyzed. Initial analysis of all known forkhead genes simultaneously using global or local alignment methods, and parsimony, likelihood or Bayesian phylogenetic methods, produced trees with inconsistent subfamily placement due to low sequence homology outside of the forkhead domain among different subfamilies. BLASTCLUST was therefore used to cluster the amino acid sequences in groups of $30 \%$ identity over $90 \%$ of their length [12]. To improve selection analysis accuracy and power, only clusters containing 10 or more sequences were included in further analyses [13]. There were five clusters, named for the majority of the sequences contained within each one, chosen for further analysis: FoxA, FoxD, FoxI, FoxO and FoxP (see Additional file 1).

\section{Alignment and Phylogenetic Analysis}

Each cluster was aligned independently using a combination of CLUSTALX1.83 [14] and CLUSTALW1.81 [15] (see Additional file 2). Amino acid sequences were aligned rather than nucleotide sequences so that gaps would not be introduced into the corresponding codons. The amino acid alignments were converted into nucleotide alignments, for phylogeny creation, utilizing the proteins' corresponding nucleotide sequences from GenBank with the program protal2dna2.0 [16]. The nucleotide alignment was then converted to nexus format with the ReadSeq2.93 [17] program for phylogenetic analysis.

MrModeltest2.2 [18] was used in conjunction with PAUP4.0b10 [19] to determine the best nucleotide substitution model for each cluster. The model chosen by the Akaike Information Criterion measure in MrModeltest was implemented in MrBayes3.1.1 [20] for each cluster. All priors were uninformative and set at default values. Each analysis was run for 1000000 generations, sampling every $100^{\text {th }}$ generation for a total of 10001 samples. A burn-in value, the number of initial samples removed from analysis, of 3000 was chosen based on previous 
analyses. The generation versus log probability plots were examined to ensure convergence was reached and that a burn-in of 3000 was appropriate. The potential scale reduction factor was also used as a measure of convergence [21].

\section{Identification of Selection Pressures}

Values of $\omega$ were estimated for each non-ambiguous codon in the alignment using the codeml program contained in the PAML3.15 package [22]. Codon site models M0, M3, M1a, M2a, M7 and M8 that estimate $\omega$, were implemented for each cluster [23-26]. Model M0 allows only one category of $\omega$ for all sites. Model M3 allowed three unconstrained $\omega$ categories, $\omega_{1}, \omega_{2}$ and $\omega_{3}$ with proportions $\mathrm{p}_{1}, \mathrm{p}_{2}$ and $\mathrm{p}_{3}=1-\mathrm{p}_{1}-\mathrm{p}_{2}$. Model M1a contains two categories of $\omega, 0<\omega_{0}<1$ and $\omega_{1}=1$ with proportions $p_{0}$ and $\mathrm{p}_{1}=1-\mathrm{p}_{0}$. Model M2a adds a third category, $\omega_{\mathrm{s}}>1$ with proportion $\mathrm{p}_{\mathrm{s}}$ such that $\mathrm{p}_{\mathrm{s}}=1-\mathrm{p}_{0}-\mathrm{p}_{1}$. Models $\mathrm{M} 7$ and M8 both contain 10 equal proportion $\omega$ categories approximated from $\beta(\mathrm{p}, \mathrm{q})$ with $0<\omega<1$ while Model M8 adds an additional $\omega$ category, $\omega_{\mathrm{s}}>1$. The proportion of sites with $\omega \sim \beta(\mathrm{p}, \mathrm{q})$ is represented by $\mathrm{p}_{0}$ and those with $\omega_{\mathrm{s}}>1$ are represented by $p_{\mathrm{s}}$ where $\mathrm{p}_{\mathrm{s}}=1-\mathrm{p}_{0}$. Each site is assigned to an $\omega$ category using a naïve empirical Bayes (NEB) (models M0, M3, M1a and M7) [27] or Bayes empirical Bayes (BEB) (models M2a and M8) [26] approach.

Codon frequencies were set as free parameters (CodonFreq $=3$ ) and ambiguous columns in the alignment were removed from the analysis. The transition/transversion ratio and branch lengths were estimated from the data using maximum likelihood methods. Two separate analyses were conducted with initial values of 0.4 and 2.0 for $\omega$ to identify and avoid local optima $[13,23]$. Each analysis was repeated once. Comparison of the results for each model using $\omega=0.4$ and $\omega=2$ and their repeats revealed that parameter estimates (In likelihood, $\mathrm{p}, \omega$ and $\beta(\mathrm{p}, \mathrm{q})$ ) for each model were identical when rounded to three decimal places. The accuracy and power of selection analysis are good if different models are tested, initial values of $\omega$ are varied and the analysis is consistent when repeated [23].

A likelihood ratio test (LRT) comparing M0 and M3 using a $\chi^{2}$ distribution with four degrees of freedom was used as a test for variation in $\omega$ among sites $[28,29]$. Two LRTs were used as a test for positive selection, M1a against $M 2 a$ and $M 7$ against $M 8$, each using a $\chi^{2}$ distribution with two degrees of freedom $[25,27]$. The LRTs were considered significant when the P-value was $\leq 0.05$. The critical values are 9.49 and 5.99 for four and two degrees of freedom respectively when $P=0.05$. A correction for multiple tests was not performed as the two LRTs for positive selection test the fit of different distributions of $\omega$ to the data and are therefore performed for robustness [30].
If positive selection occurs in only a few lineages in a tree, it may not be identified using site models, therefore branch-site model A, which allows for $\omega>1$ along a specified lineage, the foreground branch, while $\omega$ cannot be greater than one in any of the other lineages, the background branches [31] was applied. This model was implemented for lineages leading to parologous clades in the FoxA, FoxD, FoxO and FoxP clusters as positive selection is a potential evolutionary force driving subfamily paralog functional differentiation. The FoxI cluster was not examined as no lineages of interest were identified. Model A contains four classes of sites; class $0: 0<\omega_{0}<1$ and class 1: $\omega_{1}=1$, with proportions $\mathrm{p}_{0}$ and $\mathrm{p}_{1}$ respectively, for both the foreground and background branches and class $2 \mathrm{a}$ or 2b: $\omega_{2} \geq 1$ for the foreground branch with corresponding sites in the background lineage falling into class 2a: $0<\omega_{0}$ $<1$ or class $2 \mathrm{~b}: \omega_{1}=1$ site classes with proportions $\left(1-\mathrm{p}_{0^{-}}\right.$ $\left.\mathrm{p}_{1}\right) \mathrm{p}_{0} /\left(\mathrm{p}_{0}+\mathrm{p}_{1}\right)$ and $\left(1-\mathrm{p}_{0}-\mathrm{p}_{1}\right) \mathrm{p}_{1} /\left(\mathrm{p}_{0}+\mathrm{p}_{1}\right)$ respectively. All other parameters and running conditions were set as described for the site models. Model A is compared to a null model A with $\omega_{2}=1$ fixed, using a LRT and $\chi^{2}$ distribution with one degree of freedom. Statistical significance at $\alpha=0.05$ was determined after correction for multiple tests using Rom's procedure and the Bonferroni correction when multiple branches were tested in a phylogeny [32]. If significance was obtained through Rom's procedure but not the more stringent Bonferroni correction, the LRT was referred to as potentially positive. BEB is used to identify sites under positive selection if the LRT is significant and $\omega_{2}>1$.

\section{Identification of EHI Motifs}

The Engrailed Homology 1 (EH1) motif has previously been identified in many, but not all of the sequences included in this analysis [33,34]. Visual examination of the sequence alignments in conjunction with known EH1 locations suggested that there were EH1 motifs present in the sequences included in this analysis that have not been previously reported. A Perl script was written to search all of the sequences included in this analysis for the EH1 motif of the form XXaXbXXcdXX where X can be any amino acid, a can be Phe, His, Tyr or Trp, $b$ and c can be Ile, Leu or Val and d can be Glu, Phe, His, Ile, Lys, Met, Gln, Arg, Trp or Tyr $[33,35]$. Sequences with newly identified EH1 motifs are indicated in Additional file 1 and the locations of the motifs can be found in Additional file 3(A-E).

\section{Results \\ Branch-Site Analysis}

Figure 1 shows the branches that were tested for positive selection in each of the gene clusters. LRTs (Table 1) were significant for branches leading to the FoxA3 and Protostomia clades in the FoxA cluster and the FoxD2 lineage in the FoxD cluster and potentially significant for the FoxD1/ $2 / 4$ lineage in the FoxD cluster and the FoxO3 lineage in 

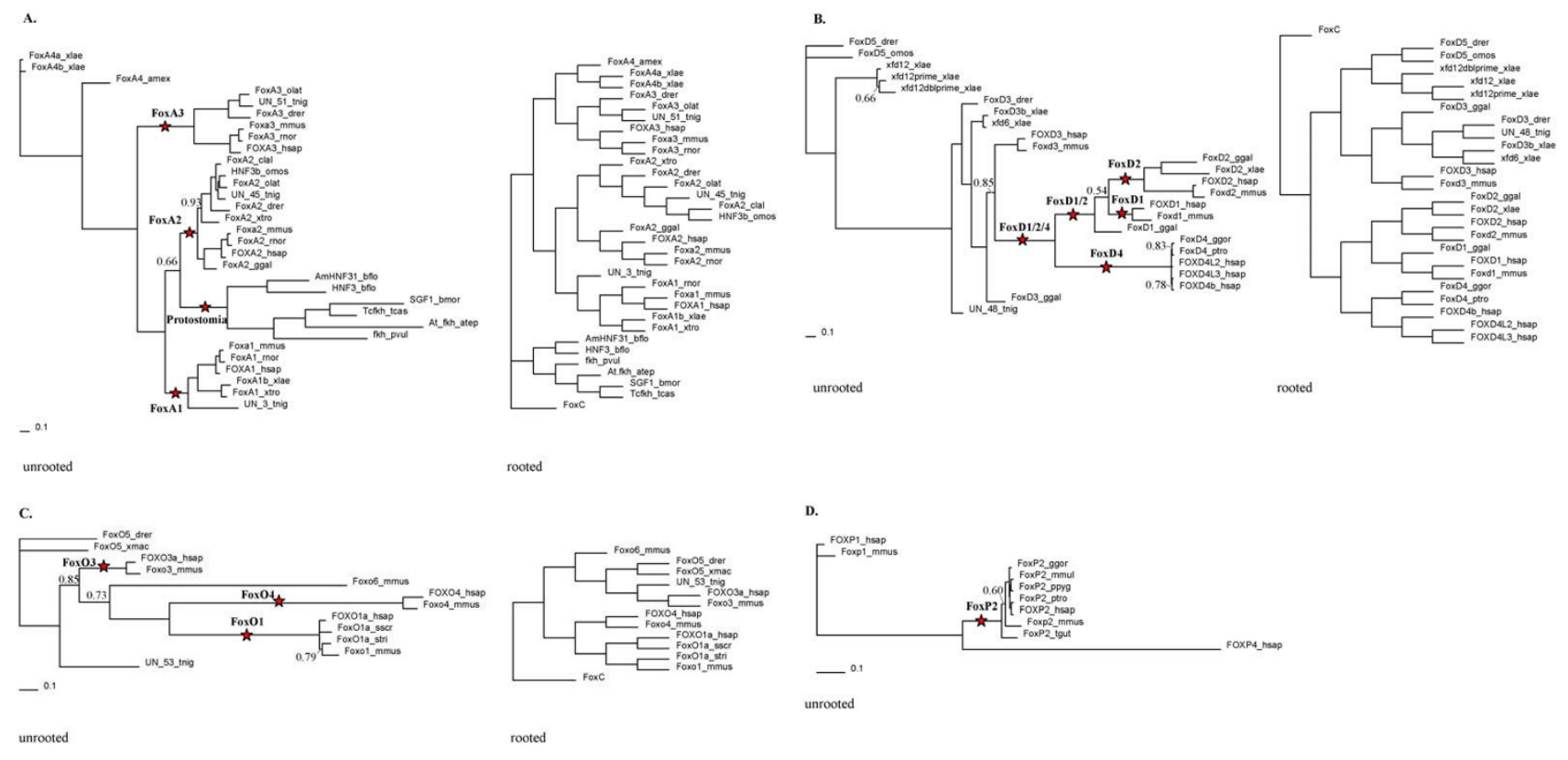

D.

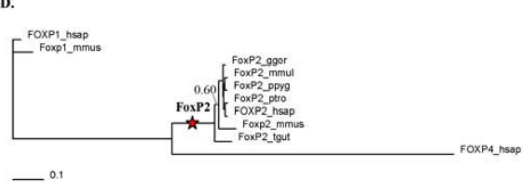

- 0.1

unrooted

Figure I

Branches tested for positive selection using branch-site models. In each phylogeny the branches tested are indicated with red stars and labels representing the clade of interest. The trees in which positive selection was identified were rooted using a set of FoxC genes as an outgroup in conjunction with neighbor joining tree creation, after branch-site analysis for clarity into evolutionary relationships. Clade credibility values less than 0.95 are indicated on the unrooted trees. The phylogenies are for the gene clusters as follows; A. FoxA B. FoxD C. FoxO D. FoxP.

the FoxO cluster, suggesting that positive selection has acted in the diversification of these paralogs from other genes in the cluster. Model A parameter estimates for lineages under positive selection are given in Table 2. Positive selection was not identified in any of the other lineages tested.
In the FoxD2 clade one positively selected site occurs between the forkhead domain and the EH1 motif in a region that has not been functionally characterized while the remaining positively selected sites identified in this lineage and that identified in the FoxD1/2/4 lineage occur within the EH1 motif as identified in the FoxD1, FoxD3

Table I: Statistical significance of the branch-site analysis LRTs after multiple corrections using Rom's procedure and the Bonferroni correction.

\begin{tabular}{|c|c|c|c|c|}
\hline Cluster & Lineage Tested & p-value of LRT & Rom's Procedure critical value & Bonferroni critical value \\
\hline \multirow[t]{4}{*}{ FoxA } & FoxAl & 0.62 & 0.0127 & 0.0125 \\
\hline & FoxA2 & 0.0634 & 0.0169 & 0.0125 \\
\hline & FoxA3 & 0.0032 & 0.025 & 0.0125 \\
\hline & Protostomia & $<0.0001$ & 0.05 & 0.0125 \\
\hline \multirow[t]{5}{*}{ FoxD } & FoxDI & 1 & 0.0102 & 0.01 \\
\hline & FoxD4 & 0.0973 & 0.0127 & 0.01 \\
\hline & FoxDI/2 & 0.0347 & 0.0169 & 0.01 \\
\hline & FoxDI/2/4 & 0.0234 & 0.025 & 0.01 \\
\hline & FoxD2 & $<0.0001$ & 0.05 & 0.01 \\
\hline \multirow[t]{3}{*}{ FoxO } & FoxO4 & 0.2628 & 0.0169 & 0.0167 \\
\hline & FoxOI & 0.109 & 0.025 & 0.0167 \\
\hline & FoxO3 & 0.0177 & 0.05 & 0.0167 \\
\hline FoxP & FoxP2 & I & 0.05 & 0.05 \\
\hline
\end{tabular}

Critical values of statistically significant results are in boldface. 
Table 2: Model A parameter estimates for significant branch-site LRTs.

\begin{tabular}{|c|c|c|c|c|c|c|}
\hline Cluster & Lineage & Site Class & Proportion & Backgound $\omega$ & Foreground $\omega$ & Positively Selected Sites* $(P \geq 0.95)$ \\
\hline \multirow[t]{8}{*}{ FoxA } & FoxA3 & 0 & 0.63948 & 0.02356 & 0.02356 & FOXA3_hsap 27P, 36G, II2P, II3L \\
\hline & & 1 & 0.330014 & I & I & \\
\hline & & $2 a$ & 0.04109 & 0.02356 & 999 & \\
\hline & & $2 b$ & 0.01929 & 1 & 999 & \\
\hline & Protostomia & 0 & 0.62704 & 0.02362 & 0.02362 & FOXAI_hsap 68Y, I59A, I99W, 234S, 237K, 242S \\
\hline & & 1 & 0.29309 & I & I & \\
\hline & & $2 a$ & 0.05443 & 0.02362 & 999 & \\
\hline & & $2 b$ & 0.02554 & 1 & 999 & \\
\hline \multirow[t]{8}{*}{ FoxD } & FoxDI/2/4 & 0 & 0.53693 & 0.02506 & 0.02506 & FOXD2_hsap R390 \\
\hline & & 1 & 0.41932 & 1 & I & \\
\hline & & $2 a$ & 0.0456 & 0.02506 & 71.85587 & \\
\hline & & $2 b$ & 0.01918 & I & 71.85587 & \\
\hline & FoxD2 & 0 & 0.51695 & 0.02526 & 0.02526 & $\begin{array}{c}\text { FOXD2_hsap E242, T386, L389, R390, Q391, G392, } \\
\text { L393, K394, T395 }\end{array}$ \\
\hline & & 1 & 0.36815 & I & 1 & \\
\hline & & $2 a$ & 0.06711 & 0.02526 & 999 & \\
\hline & & $2 b$ & 0.04779 & I & 999 & \\
\hline \multirow[t]{4}{*}{ FoxO } & FoxO3 & 0 & $0.8046 I$ & 0.04277 & 0.04277 & FOXO3_hsap S280 \\
\hline & & 1 & 0.14422 & I & I & \\
\hline & & $2 a$ & 0.04339 & 0.04277 & 10.7599 & \\
\hline & & $2 b$ & 0.00778 & I & 10.7599 & \\
\hline
\end{tabular}

* The sequence to which amino acid residues reported correspond is given for each lineage.

and FoxD5 sequences (see Additional file 3(B)). The LRT for the FoxD1/2/4 branch was potentially significant and the amino acid residues at the positively selected site identified in the FoxD1/2/4 lineage differ only in the FoxD2 lineage and are otherwise 100 percent conserved in the other sequences analyzed, therefore it is unlikely that positive selection acted along the FoxD1/2/4 lineage. The FoxD2 lineage sequences contain an EH1 motif however it was not aligned with that identified in the FoxD1, FoxD3 or FoxD5 sequences due to additional amino acids, some of which were under positive selection, found in the FoxD2 lineage. It is likely that the positive selection identified in the FoxD2 lineage within this region is due to the high conservation of the EH1 motif in the other sequences analyzed and lack of motif alignment and not due to evolutionary forces.

\section{Site Analysis}

Codon site models M0, M1a, M2a, M3, M7 and M8 were implemented in codeml for each of the six clusters and compared using likelihood ratio tests. For each cluster the M3 vs. MO LRT was significant (Table 3), indicating that one category of $\omega$ was insufficient to describe the variability in selection pressure across amino acid sites. LRTs testing for positive selection, M2a vs M1a and M8 vs M7, were also insignificant for each cluster (Table 3), therefore the amino acid changes within each cluster are neutral or under negative selection. Table 4 reports the parameter estimates for the least parameter rich model, M1a, which best describes the variation in selection pressures across sites. Graphs were constructed showing the posterior weighted $\omega$, the mean of $\omega$ over the site classes weighted by the posterior probability of each class, of each residue analyzed (Figure 2). Since ambiguous sites were removed, the residue numbers along the bottom of the graphs do not correspond to residue numbers of the analyzed sequences. Underneath each graph is a cartoon of the important regions contained in human forkhead gene(s) within that cluster. Few functional regions have been examined in human FoxA and FoxP proteins therefore functional information identified in rat and mouse protein studies has been included in the FoxA and FoxP figures respectively. The location of the forkhead domain for each human sequence was taken from the NCBI Entrez Protein [11] database record for that sequence.

\section{Discussion}

\section{Prediction of Functional and Nonfunctional Residues Using Site Analysis}

The site methods described in this paper may be used to predict functionally important residues in gene family members. If a functional domain has been identified in one member of a gene family, but not in a different member and the functional domain is under strong negative 
A

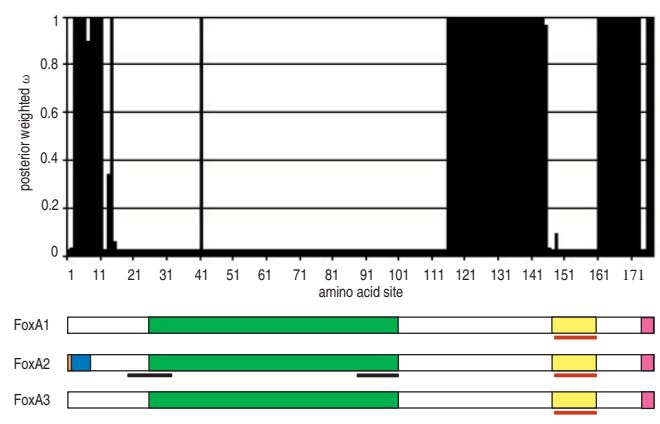

C

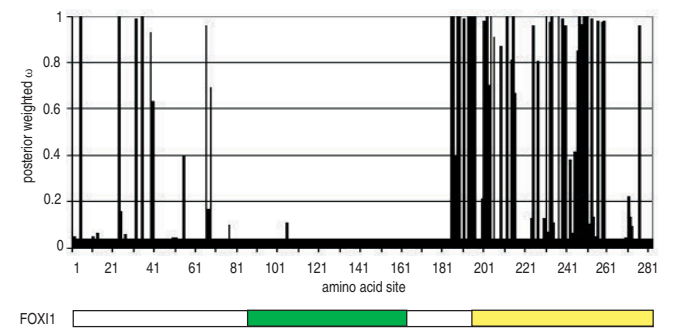

E

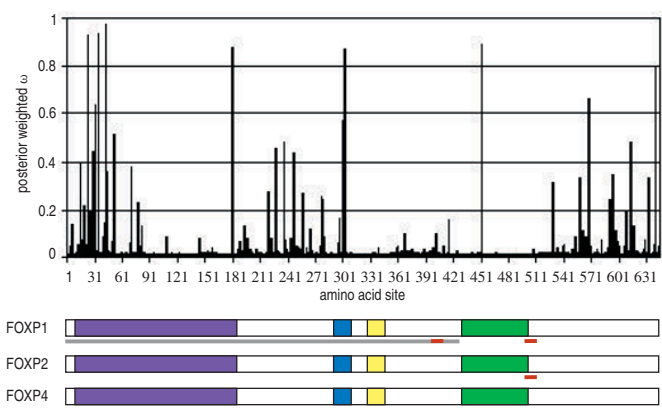

B
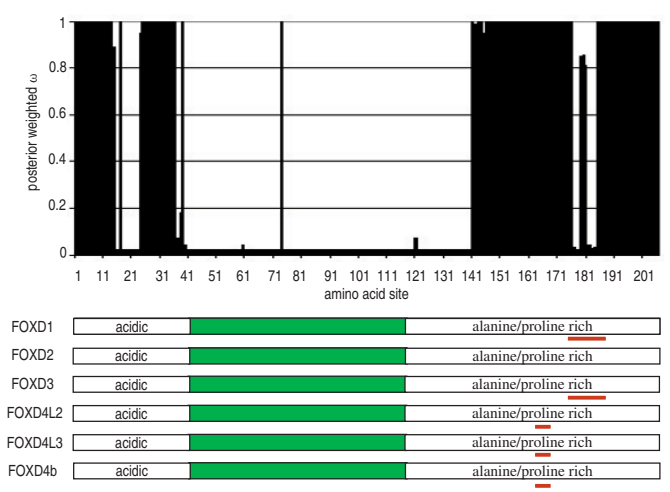

D

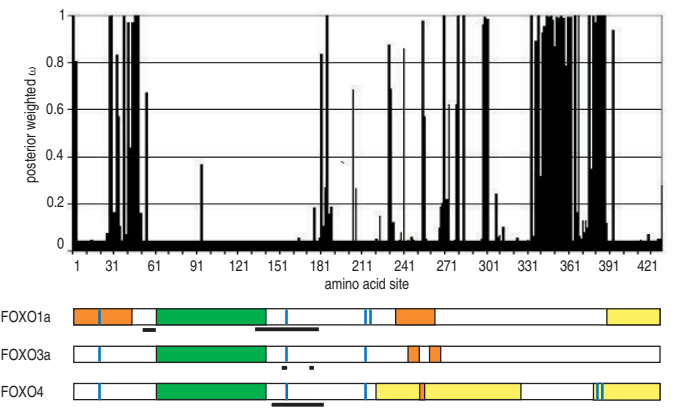

Figure 2

Selection pressures on amino acids in each of the six clusters analyzed. In each cluster the forkhead domain position was obtained from the NCBI Entrez Protein [I I] database record for that sequence (see Additional file I for accession numbers). A. FoxA cluster of 3 I sequences. Peach: conserved domain IV, site I [36,74] Blue: conserved domain V, sites 2-7 [36,74] Green: forkhead domain, sites 25-I0I Yellow: conserved domain II, sites I47-I60 [72] Pink: conserved domain III, sites I74I77 [72] Black Bar: nuclear localization signal, sites I9-32 and 88-I0I [74] Red Bar: EHI motif, sites I5I-I60 [33,34]B. FoxD cluster of 24 sequences. Green: forkhead domain, sites 42-1 19 Acidic and alanine/proline rich regions described by Ernstsson et al. 1996; Sutton et al. 1996; Ernstsson et al. 1997; Freyaldenhoven, Fried, and Wielckens 2002. Red Bar: EHI motif, sites 163-169 for all of the FOXD4s, sites 176-186 for FOXDI and FOXD3 [33,34]C. Foxl cluster of 10 sequences. Green: forkhead domain, sites 123-200 Yellow: transactivation domain, sites 196-282 [78]D. FoxO cluster of I 2 sequences. Green: forkhead domain, sites 6I-I4I Orange: nuclear export signal, sites I-43 and 235-264 for FOXOIa, sites 244-252 and 258-266 for FOXO3a, sites 250-256 for FOXO4 [42,46,47,76] Black Bar: nuclear localization signal, sites 52-60 and I 34-I80 for FOXO Ia, sites I52-I54 and I73,I74 for FOXO3a, sites I44-I83 for FOXO4 [42,45,46] Yellow: transactivation domain, sites 389-428 for FOXOIa, sites 22I-326 and 378-428 for FOXO4 [38,39] Blue Bar: phosphorylation site, sites 20, 157 and 216 for FOXOla, FOXO3a and FOXO4, additionally site 218 for FOXOIa, sites 379 and 383 for FOXO4 [44,48-52,55,56]E. FoxP cluster of 10 sequences. Purple: glutamine rich region, sites 6-I82 [58,6I,79-8I] Blue: zinc finger, sites 288-3II [58,60,6I,8I] Yellow: leucine zipper, sites 324-349 [58-6I] Green: forkhead domain, sites 434-506 Grey Bar: region involved in repression, sites I-505 [59,80] Red Bar: EHI motif, sites 398-408 and 50I-5I I for FOXPI, sites 50I-5II for FOXP2. 
selection, prediction of a similarly functioning domain may be made in the family member where a domain has not been identified. In support of this theory, the forkhead domain, which is most likely functionally active in all of the sequences analyzed, was under strong negative selection in each cluster. We were able to predict functional domains in the FoxA, FoxO and FoxP cluster sequences.

In the FoxA cluster conserved domain II has been shown to be involved in transactivation [36] and repression [37] in rat FoxA2. Since conserved domain II is entirely under strong negative selection (Figure 2A) and contained only one ambiguous column in the alignment (see Additional file 3(A)), it is likely functionally important in all of the sequences analyzed. In the FoxO cluster, a transactivation domain has been identified at the C-terminus of FOXO1a and FOXO4 $[38,39]$ while a transactivation domain has yet to be identified in FOXO3a. A portion of the C-terminal transactivation domain in FOXO4 and the entire transactivation domain in FOXO1a was under strong negative selection (Figure 2D), therefore a C-terminal transactivation domain consisting of the negatively selected residues (sites 389-428 in Figure 2D, residues 605-673 in FOXO3a) may be predicted in FOXO3a. A second, weaker, transactivation domain was identified in FOXO4 between the forkhead domain and the C-terminal transactivation domain [38]. This region is not highly conserved, although small islands of consecutive columns without gaps in the alignment that show strong negative selection, i.e. sites 315-326 in Figure 2D, may be functionally important. C-terminal deletions of PAX3FOXO1a (a fusion protein consisting of the PAX3 N-terminal region, which includes two DNA binding domains, to the C-terminal region of FOXO1a, that includes part of the forkhead domain and the C-terminal transactivation domain) that include residues within FOXO1a corresponding to the $\mathrm{FOXO} 4$ transactivation domain have also shown reduced transactivation $[40,41]$. The residues under negative selection in this region may be key to the transactivation function seen in FOXO1a and FOXO4, and residues of FOXO3a within this region may also show transactivation function. A N-terminal NES and a NLS at the N-terminus of the forkhead domain have been identified in FOXO1a [42] and were found to be under strong negative selection (Figure 2D). These regions have not been examined for NES or NLS function in FOXO3a and FOXO4. The strong negative selection of these regions suggests that a NES may be found in the N-terminus and an NLS at the N-terminus of the forkhead domain in all of the sequences analyzed. Similarly, three phosphorylation sites involved in cellular localization have been identified in FOXO1a, Ser322, Ser325 and Ser329 and have not been examined in FOXO3a and FOXO4 $[43,44]$. The Foxo6_mmus sequence was the only sequence that did not contain serines at these three positions (see Additional file 3(D)) suggesting that these serines may be functionally important in the other sequences analyzed with the exception of Foxo6_mmus. Broadly defined NLSs have also been described C-terminal to the forkhead domain in FOXO1a [45] and FOXO4 [46]. A NLS has not been defined in FOXO3a, however residues Arg248ArgArg and Lys269LysLys have been shown to function in nuclear localization [47]. This region is under strong negative selection, with the exception of one site, 181 in Figure 2D, which is under very weak negative selection, suggesting that a NLS may be found at this point in all of the sequences analyzed. Finally, there are three common phosphorylation sites among the FOXO proteins (sites 20, 157 and 216 in Figure $2 \mathrm{D}$ ) and two 14-3-3 protein binding sites (sites 1722 and 153-159 in Figure 2D) that are important in cytoplasmic/nuclear localization and therefore transactivation activity [42,45-57]. These phosphorylation and 143-3 binding sites were are all highly conserved among species and under strong negative selection suggesting functional importance in all of the sequences analyzed. Within the FoxP cluster the leucine zipper and zinc finger identified in FOXP1 and mouse Foxp1, Foxp2 and Foxp4 [58-61] were under strong negative selection suggesting that they are present in the other sequences analyzed (Figure 2E). The leucine zipper allows FoxP proteins to

Table 3: Site analysis LRT results for each cluster.

\begin{tabular}{|c|c|c|c|c|c|c|}
\hline \multirow[b]{3}{*}{ Cluster } & \multicolumn{6}{|c|}{ Models Compared } \\
\hline & \multicolumn{2}{|c|}{ M3 vs. Mo } & \multicolumn{2}{|c|}{ M2a vs. MIa } & \multicolumn{2}{|c|}{ M8 vs. M7 } \\
\hline & $2\left(\ln _{M 3}-\ln n_{M 0}\right)$ & P-value & $2\left(\ln _{M 2 a}-\ln n_{M 1 a}\right)$ & P-value & $2\left(\ln _{M 8}-\ln n_{M 7}\right)$ & P-value \\
\hline FoxA & 1446.504 & $<0.0001$ & 0 & I & 0 & I \\
\hline FoxD & 1252.774 & $<0.0001$ & 0 & I & 0 & I \\
\hline Foxl & 649.137 & $<0.0001$ & 0 & 1 & 0 & I \\
\hline FoxO & 687.901 & $<0.0001$ & 0 & I & 0 & I \\
\hline FoxP & 135.938 & $<0.0001$ & 0 & 1 & 0 & I \\
\hline
\end{tabular}

Statistically significant results at $\alpha=0.05$ are in boldface. 
Table 4: Parameter estimates of site model MIa for each cluster.

\begin{tabular}{llc}
\hline Cluster & Model & Parameter Estimates \\
\hline FoxA & Mla & $\omega_{0}=0.024 \omega_{1}=I \mathrm{P}_{0}=0.682 \mathrm{P}_{1}=0.318$ \\
FoxD & $\mathrm{Mla}$ & $\omega_{0}=0.025 \omega_{1}=I \mathrm{P}_{0}=0.555 \mathrm{P}_{1}=0.445$ \\
Foxl & $\mathrm{Mla}$ & $\omega_{0}=0.039 \omega_{1}=1 \mathrm{P}_{0}=0.832 \mathrm{P}_{1}=0.168$ \\
FoxO & $\mathrm{Mla}$ & $\omega_{0}=0.042 \omega_{1}=1 \mathrm{P}_{0}=0.839 \mathrm{P}_{1}=0.16 \mathrm{I}$ \\
FoxP & $\mathrm{Mla}$ & $\omega_{0}=0.019 \omega_{1}=1 \mathrm{P}_{0}=0.970 \mathrm{P}_{1}=0.030$ \\
\hline
\end{tabular}

form homo- and hetero-dimers $[59,60]$ and although the zinc finger function has yet to be determined, it has been suggested that it aids in dimer formation [60].

Additionally, functional domains may be predicted in regions under strong negative selection where a domain is not known to exist. For example, functionally important residues have not been identified in the N-terminus of FOXD proteins and a series of amino acids under strong negative selection is found in this region (Figure 2B). This series of negatively selected amino acids may be functionally important and forms a starting point to identifying functionally important residues outside of the forkhead domain in the FOXD proteins. Predicting functionally important residues with these methods provides a specific region of amino acids and potential domain boundaries that should be tested when searching for functional domains in vitro.

When a functional region has been identified in one gene family member, but the majority of the amino acids making up the functional region are aligned with gaps and/or are experiencing neutral changes, the region is likely not functioning in the same manner in the other sequences analyzed. Examples include conserved domains IV and V in the FoxA cluster and the transactivation domain in the FoxI cluster (Figure 2A, C, see Additional file 3(A, C)). This method identifies a region of amino acids that are less likely to be important for a specific function, which may then be examined last for functional significance when using in vitro methods.

\section{Refining Domain Boundaries Using Site Analysis}

Domain boundaries are often identified by sequence comparison to functionally related proteins or through mutagenesis experiments. When comparing sequences, it is assumed that the domain boundaries are accurately defined in the protein to which the comparison is made. Often, the boundaries of a new domain are loosely defined through mutagenesis experiments, as it is too time consuming to examine every amino acid near the suspected boundary for functional contribution. These loosely defined domains are then used by other researches in sequence comparisons to identify domains in related proteins. The methods used in this paper provide a new in silico procedure for identifying domain boundaries. For example, residues 1-50 of FOXO1a have been identified as a NES [42] however, only residues $8-32$ were under strong negative selection. This suggests that the functional domain boundaries of the N-terminal NES in FOXO1a may be redefined from residues 1 and 50 to residues 8 and 32. Molecular analysis is necessary to confirm the reallocation of domain boundaries.

The assigned boundaries of the forkhead domain vary from source to source. The NCBI Conserved Domain Database (CDD) definition of the forkhead domain, which was taken from the SMART database forkhead definition, was used in this paper. In this definition, the boundaries of the forkhead domain are defined by tertiary structure and sequence comparison of all known forkhead domains [62]. Since the C-terminal end of the forkhead domain is unstructured and variable among subfamilies [63-67], this region is excluded from the CDD forkhead domain definition even though it is involved in DNA binding [68-70]. When a new protein containing a forkhead domain is described in the literature, the forkhead domain is often identified through sequence comparison to the rat FoxA1 forkhead domain, the first forkhead domain containing protein identified in mammals [71]. The rat FoxA1 forkhead domain was broadly defined through mutational analysis [71] and then succinctly defined through sequence comparison to the rat FoxA2, FoxA3 and Drosophila Fork Head proteins $[72,73]$. When a forkhead domain is defined through sequence comparison to rat FoxA1, the N- and C-terminal domain boundaries vary within the gene family and subfamilies while the CDD definition of the forkhead domain is consistent among gene family members. The $\mathrm{N}$ - and C-terminal domain boundaries include additional amino acids when defined through sequence comparison to rat FoxA1 as compared to the CDD definition. In this analysis, a series of residues directly adjacent to the $\mathrm{N}$ - and $\mathrm{C}$-termini of the forkhead domain in each of the clusters analyzed (Figure 2) were under strong negative selection, suggesting that the forkhead domain definition should include these residues. The forkhead domain definitions supplied in the literature often accounted for some of the negatively selected sites not included in the CDD forkhead definition; however, the literature definitions either included sites that were not conserved among species, included sites with neutral changes, did not include all of the sites under negative selection and all varied in their start and stop points within subfamilies. If the $\mathrm{N}$ - and C-terminal boundaries of a domain are defined as the first and last residue respectively of a series of residues under strong negative selection, the results will be reproducible and consistent among gene family or subfamily members. 


\section{Identification of Amino Acids Involved in Paralog or Ortholog Differentiation}

The branch-site and site analysis of selection pressures on codons conducted here have identified specific amino acids responsible for differentiation of paralogs in the FoxA and FoxO clusters and orthologs in the FoxA cluster. In the FoxA cluster, the region N-terminal to the forkhead domain appears to contribute to paralog differentiation. One positively selected site identified in the FoxA3 clade occurs within conserved domain IV and one positively selected site identified in the Protostomia lineage occurs within conserved domain $\mathrm{V}$ as both domains are defined in FoxA2 [74] (see Additional file 3(A)). Overall conserved domains IV and $\mathrm{V}$, which have been shown to play a role in transactivation in FoxA2 proteins [74], are not well conserved in the FoxA3 or Protostomia proteins as compared to the FoxA1 and FoxA2 proteins as the majority of the residues making up these domains were not analyzed due to gaps in the alignment and those that were examined by site analysis show variability in selection pressure with most of the sites, 5/7, having experienced neutral changes (Figure 2A). Additional sites under positive selection $\mathrm{N}$-terminal to the forkhead domain were also identified through branch-site analysis in the FoxA3 and Protostomia lineages (see Additional file 3(A)). Two of these sites in the FoxA3 lineage occur in a nuclear localization signal (NLS) that was broadly defined in rat FoxA2 [74] while the other positively selected sites are found in regions uncharacterized in any FoxA protein. FoxA1 and FoxA2 have more similar expression patterns and functions during development and metabolism as compared to the FoxA3 proteins (reviewed by [75]). This evidence in conjunction with the positive selection identified here suggests that the $\mathrm{N}$-terminal region of sequences not included in the FoxA1 or FoxA2 clades have evolved to differentiate these proteins from the FoxA1 and FoxA2 proteins while the sequences were conserved in the FoxA1 and FoxA2 proteins leading to overlapping expression and function.

Conserved domain III, which has been shown to function in transactivation in rat FoxA2 [36] contained many ambiguous sites in the FoxA alignment (see Additional file 3(A)) due to sequences from the Protostomia lineage and variations in selection pressure were observed in the four sites, through site analysis, that did contain amino acids from these species (Figure 2A). This suggests that conserved domain III is important for FoxA function in the Deuterostomia but not in the Protostomia and that the FoxA genes in the two lineages have evolved to perform species specific functions. Therefore the presence of conserved domain III may differentiate FoxA orthologs between the Protostomia and Deuterostomia lineages.
In the FoxO cluster, the NES(s) located between the forkhead domain and the C-terminus in the FOXO1a, FOXO3a and FOXO4 sequences $[42,46,47,76]$ are not highly conserved among the FoxO family members as their alignment was not well defined, only three sites, 250-252, in Figure 2D contain NES residues from each of the three human FOXO proteins examined and some residues have experienced neutral changes as demonstrated by site analysis. These NES(s) may be used to differentiate FoxO paralogs.

Only one site was found to be under positive selection in the FoxO3 lineage during branch-site analysis and the LRT was potentially significant. This residue is found in a region important for nuclear localization, C-terminal to the forkhead domain (see Additional file 3(C)). The amino acid located at the positively selected site is serine in the FoxO3 sequences while it is glycine, alanine or aspartic acid in the other sequences analyzed. The presence of serine at this position may be important for regulation of the FoxO3 proteins by phosphorylation and this regulation may be different from the other FoxO sequences analyzed. Molecular testing is required to validate this hypothesis.

In summary, residues that differentiate paralogs were identified in the FoxA and FoxO clusters while residues that differentiate orthologs were also identified in the FoxA cluster. This information provided insights into the evolution of these two subfamilies. Within the FoxD, FoxI, and FoxP clusters, residues that differentiate orthologs or paralogs were unidentifiable due to lack of functional information (FoxD and FoxI clusters only) and overall negative selection in the identified domains.

\section{Subfamily Evolution}

Forkhead subfamilies are defined by their homology in the forkhead domain alone. Here we analyzed the entire coding regions of forkhead proteins and found that the subfamily structures were maintained after sequence analysis with BLASTCLUST. Our site analysis also demonstrated distinct regions of homology outside the forkhead domain in each of the clusters analyzed, further supporting the subfamily member evolutionary relationships defined by the forkhead domain alone.

The patterns of strong negative and neutral selection observed through site analysis in each of the clusters and through branch-site analysis along the majority of the lineages tested, indicate that after gene duplication, rapid differentiation of paralogs through codon changes and subsequent maintenance, negative selection, of these changes has occurred. The lack of positive selection observed through site analysis indicates that the functions 
of forkhead gene family members as we see them today have been determined and fixed in the species analyzed. However, the positive selection observed along select lineages in the FoxA and FoxO cluster indicate more recent or observable continuing functional divergence. While the majority of studies that have used these methods focus only on positive selection, a few involving transcription factor gene families have discussed negative selection as well. Our results are similar to those seen in a comparable analysis of HOX7 where heterogeneous selection pressures but not positive selection were observed during site analysis and positive selection was observed on a single branch separating paralogs during branch-site analysis [77]. These types of analysis of gene families that were originally defined by a common functional motif may confirm or refute the family relationships and provide insights into their evolutionary development. If positive selection is observed it suggests that the evolutionary changes are ortholog or paralog differentiating while negative selection indicates that the protein function is conserved among species.

\section{Forkhead Domain Evolution}

As forkhead subfamilies are defined by and forkhead gene function is reliant on the forkhead domain, identification of selection pressures acting on codons within the domain provides insights into the functional evolution of subfamilies and their paralogs. In each of the subfamilies, the majority of the residues in the forkhead domain were under strong negative selection (Figure 2) consistent with the general consensus that the domain is highly conserved and important for proper gene function. More interestingly, sites under positive selection and neutral changes were observed in the forkhead domain in some subfamilies and these provide insights into the evolutionary differentiation of forkhead genes.

In the FoxA cluster Protostomia lineage a number of residues under positive selection were found in the forkhead domain through branch-site analysis. These residues are located within helix $2, \beta$-sheet 2 and wing 1 as defined by the crystal structure of FoxA3 [63] (Figure 3, see Additional file $3(\mathrm{~A})$ ). The residues corresponding to the positively selected sites in the Protostomia lineage are 100 percent conserved among the other sequences analyzed. It is possible that these changes in amino acid composition of the forkhead domain alter the domain configuration thus allowing for different target binding and/or regulation of FoxA genes in the Protostomia as compared to the Deuterostomia. It is interesting to note that to date, in most Protostomia only one FoxA class gene has been identified while in the Deuterostomia, multiple FoxA class genes have been found. If FoxA targets are similar in the Protostomia and Deuterostomia lineages, the altera-

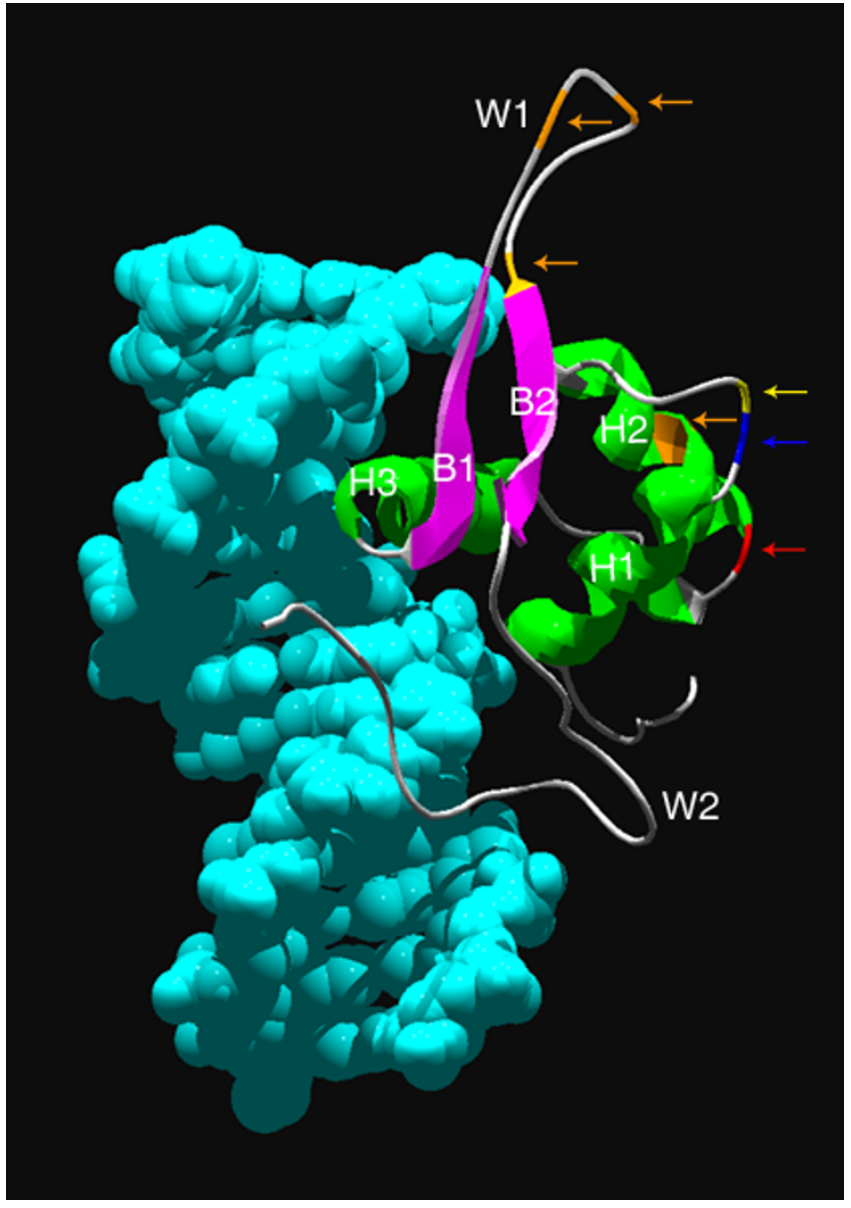

Figure 3

Residues experiencing neutral changes and positive selection in the forkhead domain. The forkhead domain of FOXA3 is shown bound to DNA. Residues with neutral changes identified in the FoxA (blue), FoxD (red) and FoxP (yellow) clusters and residues under positive selection identified in the Protostomia lineage of the FoxA cluster (orange) are highlighted and indicated with arrows. Alpha helices I, 2 and 3 , beta sheets $I$ and 2 , and wings $I$ and 2 are denoted $\mathrm{HI}, \mathrm{H} 2, \mathrm{H} 3, \mathrm{BI}, \mathrm{B} 2, \mathrm{WI}$ and $\mathrm{W} 2$ respectively.

tions in the forkhead domain of Protostomia FoxA may allow these single proteins to perform the same function that require multiple FoxA proteins in the Deuterostomia. This theory is further supported by the differences observed in the N-terminal region of the Protostomia FoxA and in conserved domain III as compared to the Deuterostomia discussed earlier.

One residue within the forkhead domain was experiencing neutral changes in the FoxA, FoxD and FoxP clusters (Figures 2A (site 41), 2B (site 74), 2E (site 451)). The locations of the residues with neutral changes are shown on the FoxA3 crystal structure in Figure 3. The sites experienc- 
ing neutral changes identified in the FoxA and FoxP clusters were found at the C-terminus of alpha helix 1 while the site experiencing neutral changes in the FoxD cluster was located near the C-terminus of alpha helix 2. Neutral changes at a site imply that any amino acid may be present at that site and amino acid changes will not affect protein function. In support of this theory, mutation of the site corresponding to the neutral site identified in the FoxD cluster in rat FoxA3 from aspartate to lysine did not affect DNA binding [68]. The sites with neutral changes identified in the FoxA, FoxD and FoxP clusters and the corresponding sites in other Fox proteins have not been associated with point mutations causing human disease and have not been shown to contact DNA during DNA binding. The NCBI Entrez SNP database [11], Build 126, was used to determine if the sites with neutral changes have naturally occurring single nucleotide polymorphisms in any of the forkhead genes found in humans. Only one forkhead gene, FOXD4, has a known SNP at a location corresponding to one of the sites with neutral changes. The SNP identified in FOXD4 corresponds to the neutrally changed site identified in the FoxD proteins and is either aspartate or glycine. It would be interesting to determine if amino acid changes at these sites affect forkhead domain function and if the neutrally changed sites are common to the forkhead domain or specific to the subfamilies in which they were identified.

The variations from negative selection in the forkhead domain identified here may account for differences in subfamily and paralog function that are not explained by differences in timing or location of expression or other functional regions in the proteins.

\section{Conclusion}

This analysis has provided insights into forkhead gene family and subfamily evolution. Through identification of selection pressures we provided evidence for the functional and evolutionary importance of amino acid differences in paralogs and orthologs of FOX subfamilies. Our work has also supported the forkhead subfamily structure and identified a pattern of evolution in the family. Additionally, our analyses allowed evaluation and extension of domain structural and positional information between gene family members. Future in vitro studies may use this information as a starting point or for refinement of protein functional analysis.

\section{Authors' contributions}

CF participated in study design, carried out all experiments and drafted the manuscript. BR and MW conceived of the study and participated in its design. MW assisted in manuscript preparation.

\section{Additional material}

\author{
Additional file 1 \\ Composition of the sequence clusters analyzed. This table gives the \\ sequence composition of the clusters analyzed and notes sequences in \\ which EH1 motifs were newly identified. \\ Click here for file \\ [http://www.biomedcentral.com/content/supplementary/1471- \\ 2148-8-261-S1.pdf]
}

\section{Additional file 2}

Alignment procedure with ClustalX and ClustalW. The procedure used to create multiple sequence alignments is provided in this file.

Click here for file

[http://www.biomedcentral.com/content/supplementary/14712148-8-261-S2.pdf]

\section{Additional file 3}

Amino acid alignments. The amino acid alignment of each of the clusters analyzed (A. FoxA, B. FoxD, C. FoxI, D. FoxO and E. FoxP) with regions of interest highlighted is shown here.

Click here for file

[http://www.biomedcentral.com/content/supplementary/1471-

2148-8-261-S3.pdf]

\section{Acknowledgements}

This work was supported by the Alberta Heritage Foundation for Medical Research, the Natural Sciences and Engineering Research Council of Canada and the Canadian Institutes of Health Research.

\section{References}

I. Lines MA, Kozlowski K, Walter MA: Molecular genetics of Axenfeld-Rieger malformations. Hum Mol Genet 2002, II(10): II77-II84

2. Fang J, Dagenais SL, Erickson RP, Arlt MF, Glynn MW, Gorski JL, Seaver LH, Glover TW: Mutations in FOXC2 (MFH-I), a forkhead family transcription factor are responsible for the hereditary lymphedema-distichiasis syndrome. Am J Hum Genet 2000, 67:1382-1388.

3. Lai CSL, Fisher SE, Hurst JA, Vargha-Khadem F, Monaco AP: A forkhead-domain gene is mutated in a severe speech and language disorder. Nature 200I, 4I3(6855):5I9-523.

4. Galili N, Davis RJ, Fredericks WJ, Mukhopadhyay S, Rausher FJ 3rd, Emanual BS, Rovera G, Barr FG: Fusion of a fork head domain gene to PAX3 in the solid tumour alveolar rhabdomyosarcoma. Nature Genetics 1993, 5(3):230-235.

5. Hillion J, Le Coniat M, Jonveaux P, Berger R, Bernard OA: AF6q2I, a novel partner of the MLL gene in $\mathrm{t}(6 ; \mathrm{I} I)(\mathrm{q} 2 \mathrm{I} ; \mathrm{q} 23)$, defines a forkhead transcriptional factor subfamily. Blood 1997, 90(9):37|4-37|9.

6. Lin L, Miller CT, Contreras JI, Prescott MS, Dagenais SL, Wu R, Yee J, Orringer MB, Misek DE, Hanash SM, Glover TW, Beer DG: The hepatocyte nuclear factor 3 alpha gene, HNF3alpha (FOXAI), on chromosome band $14 \mathrm{q} / 3$ is amplified and overexpressed in esophageal and lung adenocarcinomas. Cancer Res 2002, 62( ( 8):5273-5279.

7. Parry P, Wei Y, Evans G: Cloning and characterization of the $t(X ; I I)$ breakpoint from a leukemic cell line identify a new member of the forkhead gene family. Genes Chromosomes \& Cancer 1994, I I (2):79-84.

8. Kaestner KH, Knöchel W, Martínez DE: Unified nomenclature for the winged helix/forkhead transcription factors. Genes Dev 2000, 14: $142-146$. 
9. Geer LY, Domrachev M, Lipman DJ, Bryant SH: CDART: Protein homology by domain architecture. Genome Research 2002 , 12:1619-1623.

10. Marchler-Bauer A, Anderson JB, Cherukuri PF, DeWeese-Scott C Geer LY, Gwadz M, He S, Hurwitz DI, Jackson JD, Ke Z, Lanczycki CJ, A LC, Liu C, Lu F, Marchler GH, Mullokandov M, Shoemaker BA, Simonyan V, Song JS, Thiessen PA, Yamashita RA, Yin J], Zhang D, Bryant SH: CDD: a Conserved Domain Database for protein classification. Nucleic Acids Research 2005, 33:DI92-DI96.

II. Wheeler DL, Barrett T, Benson DA, Bryant SH, Canese K Chetvernin V, Church DM, DiCuccio M, Edgar R, Federhen S, Geer LY, Helmberg W, Kapustin Y, Kenton DL, Khovayko O, Lipman DJ, Madden TL, Maglott DR, Ostell J, Pruitt KD, Schuler GD, Schriml LM, Sequeira E, Sherry ST, Sirotkin K, Souvorov A, Starchenko G, Suzek TO, Tatusov R, Tatusova TA, Wagner L, Yaschenko E: Database resources of the National Center for Biotechnology Information. Nucleic Acids Res 2006:D I73-180.

12. Altschul SF, Madden TL, Schaffer AA, Zhang J, Zhang Z, Miller W, Lipman DJ: Gapped BLAST and PSI-BLAST: a new generation of protein database search programs. Nucleic Acids Res 1997, 25(I 7):3389-3402.

13. Anisimova M, Bielawski JP, Yang Z: Accuracy and power of Bayes prediction of amino acid sites under positive selection. Mol Biol Evol 2002, 19(6):950-958.

14. Thompson JD, Gibson TJ, Plewniak F, Jeanmougin F, Higgins DG: The CLUSTAL_ $X$ windows interface: flexible strategies for multiple sequence alignment aided by quality analysis tools. Nucleic Acids Res 1997, 25(24):4876-4882.

15. Thompson JD, Higgins DG, Gibson TJ: CLUSTAL W: improving the sensitivity of progressive multiple sequence alignment through sequence weighting, position-specific gap penalties and weight matrix choice. Nucleic Acids Res 1994 22(22):4673-4680.

16. Letondal C, Schuerer K: protal2dna. 2.0th edition. Paris, France: Pasteur Institute

17. Gilbert DG: Readseq version 2, an improved biosequence conversion tool, written in the java language. 2.93th edition. Bloomington, Indiana: Bionet Software; 1999.

18. Nylander JAA: MrModeltest 2.0. 2.2th edition. Uppsala, Sweden: Program distributed by the author; 2004

19. Swofford DL: PAUP*: phylogenetic analysis using parsimony (* and other methods). $4.0 \mathrm{bl} 0$ edition. Sunderland, Massachusetts, USA: Sinauer Associates; 2002.

20. Ronquist F, Huelsenbeck JP: MrBayes 3: Bayesian phylogenetic inference under mixed models. Bioinformatics 2003 , I 9(1 2): I 572-I574.

21. Gelman A, Rubin DB: Inference from iterative simulation using multiple sequences. Statistical Science 1992, 7(4):457-5 I I.

22. Yang Z: PAML: a program package for phylogenetic analysis by maximum likelihood. Computer Applications in the Biosciences I997, I3(5):555-556.

23. Wong WSW, Yang Z, Goldman N, Nielsen R: Accuracy and power of statistical methods for detecting adaptive evolution in protein coding sequences and for identifying positively selected sites. Genetics 2004, I 68: |04|-105|

24. Yang Z, Nielsen R, Hasegawa M: Models of amino acid substitution and applications to mitochondrial protein evolution. Mol Biol Evol I 998, I5(12): I600-I6II |

25. Yang Z, Nielsen R, Goldman N, Pedersen A-MK: Codon-substitution models for heterogeneous selection pressure at amino acid sites. Genetics 2000, I 55:43 I-449.

26. Yang Z, Wong WSW, Nielsen R: Bayes empirical Bayes inference of amino acid sites under positive selection. Mol Biol Evol 2005, 22(4): I 107-III8.

27. Nielsen R, Yang Z: Likelihood models for detecting positively selected amino acid sites and applications to the HIV-I envelope gene. Genetics 1998, 148:929-936.

28. Anisimova M, Bielawski JP, Yang Z: Accuracy and power of the likelihood ratio test in detecting adaptive molecular evolution. Mol Biol Evol 200I, I 8(8): I585-I592.

29. Yang Z, Swanson WJ, Vacquier VD: Maximum-likelihood analysis of molecular adaptation in abalone sperm lysin reveals variable selective pressures among lineages and sites. Mol Biol Evol 2000, I 7( I 0): | 446-| 455 .
30. Bonferroni Correction [http://gsf.gc.ucdavis.edu/view topic.php? $f=\mid$ \& $t=\mid 484 \& p=2996 \&$ hilit=bonferroni+correc tion\&sid=fec34f5a576643cc276fd52c7add4 I I 6\#p2996]

3I. Zhang J, Nielsen R, Yang Z: Evaluation of an improved branchsite likelihood method for detecting positive selection at the molecular level. Mol Biol Evol 2005, 22(I 2):2472-2479.

32. Anisimova M, Yang Z: Multiple hypothesis testing to detect lineages under positive selection that affects only a few sites. Mol Biol Evol 2007, 24(5): I 2 I9-I 228.

33. Copley RR: The EHI motif in metazoan transcription factors. BMC Genomics 2005, 6:169.

34. Yaklichkin S, Vekker A, Stayrook S, Lewis M, Kessler DS: Prevalence of the EHI Groucho interaction motif in the metazoan Fox family of transcriptional regulators. BMC Genomics 2007, 8:201.

35. Smith ST, Jaynes JB: A conserved region of engrailed, shared among all en-, gsc-, NkI-, Nk2- and msh-class homeoproteins, mediates active transcriptional repression in vivo. Development 1996, I 22(I 0):3|4|-3|50.

36. Pani L, Overdier DG, Porcella A, Qian X, Lai E, Costa RH: Hepatocyte nuclear factor $3 \beta$ contains two transcriptional activation domains, one of which is novel and conserved with the Drosophila fork head protein. Mol Cell Biol 1992, I 2(9):3723-3732.

37. Wang JC, Waltner-Law M, Yamada K, Osawa H, Stifani S, Granner DK: Transducin-like enhancer of split proteins, the human homologs of Drosophila groucho, interact with hepatic nuclear factor 3beta. J Biol Chem 2000, 275(24): I84 I8-18423.

38. So CW, Cleary ML: MLL-AFX requires the transcriptional effector domains of AFX to transform myeloid progenitors and transdominantly interfere with forkhead protein function. Mol Cell Biol 2002, 22( I 8):6542-6552.

39. Sublett JE, Jeon IS, Shapiro DN: The alveolar rhabdomyosarcoma PAX3/FKHR fusion protein is a transcriptional activator. Oncogene 1995, I I(3):545-552.

40. Kempf BE, Vogt PK: A genetic analysis of PAX3-FKHR, the oncogene of alveolar rhabdomyosarcoma. Cell Growth Differ 1999, I0(I 2):813-818.

4I. Lam PY, Sublett JE, Hollenbach AD, Roussel MF: The oncogenic potential of the Pax3-FKHR fusion protein requires the $\operatorname{Pax} 3$ homeodomain recognition helix but not the $P$ ax 3 paired-box DNA binding domain. Mol Cell Biol 1999, I 9(I):594-60I.

42. Zhao X, Gan L, Pan H, Kan D, Majeski M, Adam SA, Unterman TG: Multiple elements regulate nuclear/cytoplasmic shuttling of FOXOI: characterization of phosphorylation- and 14-3-3dependent and -independent mechanisms. Biochem / 2004, 378(Pt 3):839-849.

43. Rena G, Woods YL, Prescott AR, Peggie M, Unterman TG, Williams MR, Cohen P: Two novel phosphorylation sites on FKHR that are critical for its nuclear exclusion. EMBO J 2002, 2 I(9):2263-227I.

44. Woods YL, Rena G, Morrice N, Barthel A, Becker W, Guo S, Unterman TG, Cohen P: The kinase DYRKIA phosphorylates the transcription factor FKHR at Ser329 in vitro, a novel in vivo phosphorylation site. Biochem J 200 I, 355(Pt 3):597-607.

45. Zhang X, Gan L, Pan H, Guo S, He X, Olson ST, Mesecar A, Adam S, Unterman TG: Phosphorylation of serine 256 suppresses transactivation by FKHR (FOXOI) by multiple mechanisms. Direct and indirect effects on nuclear/cytoplasmic shuttling and DNA binding. I Biol Chem 2002, 277(47):45276-45284.

46. Brownawell AM, Kops GJ, Macara IG, Burgering BM: Inhibition of nuclear import by protein kinase $B$ (Akt) regulates the subcellular distribution and activity of the forkhead transcription factor AFX. Mol Cell Biol 200I, 2 I (I 0):3534-3546

47. Brunet A, Kanai F, Stehn J, Xu J, Sarbassova D, Frangioni JV, Dalal SN DeCaprio JA, Greenberg ME, Yaffe MB: I4-3-3 transits to the nucleus and participates in dynamic nucleocytoplasmic transport. J Cell Biol 2002, I 56(5):817-828.

48. Nakae J, Park BC, Accili D: Insulin stimulates phosphorylation of the forkhead transcription factor FKHR on serine 253 through a Wortmannin-sensitive pathway. I Biol Chem 1999, 274(23): I 5982-I5985

49. Rena G, Guo S, Cichy SC, Unterman TG, Cohen P: Phosphorylation of the transcription factor forkhead family member FKHR by protein kinase B. J Biol Chem 1999, 274(24): $|7| 79-|7| 83$ 
50. Brunet A, Bonni A, Zigmond MJ, Lin MZ, Juo P, Hu LS, Anderson M], Arden KC, Blenis J, Greenberg ME: Akt promotes cell survival by phosphorylating and inhibiting a Forkhead transcription factor. Cell 1999, 96(6):857-868.

5I. Brunet A, Park J, Tran H, Hu LS, Hemmings BA, Greenberg ME: Protein kinase SGK mediates survival signals by phosphorylating the forkhead transcription factor FKHRLI (FOXO3a). Mol Cell Biol 200I, 2 I (3):952-965.

52. Kops GJ, de Ruiter ND, De Vries-Smits AM, Powell DR, Bos JL, Burgering BM: Direct control of the Forkhead transcription factor AFX by protein kinase B. Nature 1999, 398(6728):630-634.

53. Obsil T, Ghirlando R, Anderson DE, Hickman AB, Dyda F: Two I4 3-3 binding motifs are required for stable association of Forkhead transcription factor FOXO4 with 14-3-3 proteins and inhibition of DNA binding. Biochemistry 2003, 42(5 I): I5264-I5272.

54. Rena G, Prescott AR, Guo S, Cohen P, Unterman TG: Roles of the forkhead in rhabdomyosarcoma (FKHR) phosphorylation sites in regulating 14-3-3 binding, transactivation and nuclear targetting. Biochem J 200I, 354(Pt 3):605-6I2

55. Takaishi $H$, Konishi H, Matsuzaki H, Ono Y, Shirai $Y$, Saito N, Kitamura T, Ogawa W, Kasuga M, Kikkawa U, Nishizuka Y: Regulation of nuclear translocation of forkhead transcription factor AFX by protein kinase B. Proc Natl Acad Sci USA 1999, 96(2I): II836-II84I.

56. Tang ED, Nunez G, Barr FG, Guan KL: Negative regulation of the forkhead transcription factor FKHR by Akt. J Biol Chem 1999, 274(24): I674I-I6746.

57. Mazumdar A, Kumar R: Estrogen regulation of Pak I and FKHR pathways in breast cancer cells. FEBS Lett 2003, 535(I-3):6-10.

58. Banham AH, Beasley N, Campo E, Fernandez PL, Fidler C, Gatter K, Jones M, Mason DY, Prime JE, Trougouboff P, Wood K, Cordell JL: The FOXPI winged helix transcription factor is a novel candidate tumor suppressor gene on chromosome $3 \mathrm{p}$. Cancer Res 200I, 6 I (24):8820-8829.

59. Li S, Weidenfeld J, Morrisey EE: Transcriptional and DNA binding activity of the FoxpI/2/4 family is modulated by heterotypic and homotypic protein interactions. Mol Cell Biol 2004, 24(2):809-822.

60. Wang B, Lin D, Li C, Tucker P: Multiple domains define the expression and regulatory properties of Foxpl forkhead transcriptional repressors. I Biol Chem 2003, 278(27):24259-24268.

6I. Teufel A, Wong EA, Mukhopadhyay M, Malik N, Westphal H: FoxP4, a novel forkhead transcription factor. Biochim Biophys Acta 2003 , | 627(2-3): | $47-152$

62. Schultz J, Copley RR, Doerks T, Ponting CP, Bork P: SMART: a web-based tool for the study of genetically mobile domains. Nucleic Acids Res 2000, 28(I):231-234.

63. Clark KL, Halay ED, Lai E, Burley SK: Co-crystal structure of the HNF-3/fork head DNA-recognition motif resembles histone H5. Nature 1993, 364(6436):412-420.

64. Marsden I, Jin C, Liao X: Structural changes in the region directly adjacent to the DNA-binding helix highlight a possible mechanism to explain the observed changes in the sequence-specific binding of winged helix proteins. J Mol Biol 1998, 278(2):293-299.

65. Stroud JC, Wu Y, Bates DL, Han A, Nowick K, Paabo S, Tong H, Chen L: Structure of the forkhead domain of FOXP2 bound to DNA. Structure 2006, I4(I): 159-166.

66. van Dongen MJ, Cederberg A, Carlsson P, Enerback S, Wikstrom M Solution structure and dynamics of the DNA-binding domain of the adipocyte-transcription factor FREAC-II. J Mol Biol 2000, 296(2):35I-359.

67. Weigelt J, Climent I, Dahlman-Wright K, Wikstrom M: IH, I3C and I $5 \mathrm{~N}$ resonance assignments of the DNA binding domain of the human forkhead transcription factor AFX. J Biomol NMR 2000, I7(2): $181-182$

68. Clevidence DE, Overdier DG, Tao W, Qian X, Pani L, Lai E, Costa $\mathrm{RH}$ : Identification of nine tissue-specific transcription factors of the hepatocyte nuclear factor 3/forkhead DNA-bindingdomain family. Proc Natl Acad Sci USA 1993, 90(9):3948-3952.

69. Pierrou S, Hellavist M, Samuelsson L, Enerback S, Carlsson P: Cloning and characterization of seven human forkhead proteins: binding site specificity and DNA bending. EMBO J 1994 I3(20):5002-50|2.
70. Shiyanova $T$, Liao $X$ : The dissociation rate of a winged helix protein-DNA complex is influenced by non-DNA contact residues. Arch Biochem Biophys 1999, 362(2):356-362.

7I. Lai E, Prezioso VR, Smith E, Litvin O, Costa RH, Darnell JE Jr: HNF$3 A$, a hepatocyte-enriched transcription factor of novel structure is regulated transcriptionally. Genes Dev 1990 4(8): | 427-I 436.

72. Lai E, Prezioso VR, Tao WF, Chen WS, Darnell JE Jr: Hepatocyte nuclear factor 3 alpha belongs to a gene family in mammals that is homologous to the Drosophila homeotic gene fork head. Genes Dev 199I, 5(3):416-427.

73. Weigel $D$, jackle $H$ : The fork head domain: a novel DNA binding motif of eukaryotic transcription factors? Cell 1990, 63(3):455-456

74. Qian X, Costa RH: Analysis of hepatocyte nuclear factor-3 protein domains required for transcriptional activation and nuclear targeting. Nucleic Acids Res 1995, 23(7): I | 84- I I 1 .

75. Friedman JR, Kaestner KH: The Foxa family of transcription factors in development and metabolism. Cell Mol Life Sci 2006, 63(19-20):23|7-2328.

76. Biggs WH 3rd, Meisenhelder J, Hunter T, Cavenee WK, Arden KC: Protein kinase B/Akt-mediated phosphorylation promotes nuclear exclusion of the winged helix transcription factor FKHRI. Proc Natl Acad Sci USA 1999, 96( I3):742I-7426.

77. Fares MA, Bezemer D, Moya A, Marin I: Selection on coding regions determined Hox7 genes evolution. Mol Biol Evol 2003, 20( (I2):2104-2III

78. Overdier DG, Ye H, Peterson RS, Clevidence DE, Costa RH: The winged helix transcriptional activator HFH-3 is expressed in the distal tubules of embryonic and adult mouse kidney. J Biol Chem 1997, 272(21): I3725-13730.

79. Lai CS, Fisher SE, Hurst JA, Vargha-Khadem F, Monaco AP: A forkhead-domain gene is mutated in a severe speech and language disorder. Nature 200I, 4I3(6855):5I9-523.

80. Shi C, Zhang X, Chen Z, Sulaiman K, Feinberg MW, Ballantyne CM, Jain MK, Simon DI: Integrin engagement regulates monocyte differentiation through the forkhead transcription factor Foxp I. J Clin Invest 2004, I I 4(3):408-4I8.

8I. Shu W, Yang H, Zhang L, Lu MM, Morrisey EE: Characterization of a new subfamily of winged-helix/forkhead (Fox) genes that are expressed in the lung and act as transcriptional repressors. J Biol Chem 200I, 276(29):27488-27497.

Publish with Biomed Central and every scientist can read your work free of charge

"BioMed Central will be the most significant development for disseminating the results of biomedical research in our lifetime. "

Sir Paul Nurse, Cancer Research UK

Your research papers will be:

- available free of charge to the entire biomedical community

- peer reviewed and published immediately upon acceptance

- cited in PubMed and archived on PubMed Centra

- yours - you keep the copyright 